\title{
Management of hydrocephalus in pediatric patients with posterior fossa tumors: the role of endoscopic third ventriculostomy
}

\section{Christian Sainte-Rose, M.D., Giuseppe Cinalli, M.D., Franck E. Roux, M.D., Wirginia Maixner,} F.R.A.C.S., Paul D. Chumas, F.R.C.S.(SN), Maheir Mansour, M.D., Alexandre Carpentier, M.D., Marie Bourgeois M.D., Michel Zerah, M.D., Alain Pierre-Kahn, M.D., and Dominique Renier, M.D.

Service de Neurochirurgie, Hopital Necker-Enfants Malades, Paris, France; Divisione di Neurochirurgia Pediatrica, Ospedale "Santobono," Naples, Italy, and Department of Neurosurgery, The General Infirmary at Leeds, Leeds, United Kingdom

The authors conducted a study to evaluate the effectiveness of endoscopically guided third ventriculostomy in the pre- and postoperative management of hydrocephalus in pediatric patients who harbored posterior fossa tumors.

Between October 1, 1993, and December 31, 1997, a total of 206 consecutive children with posterior fossa tumors underwent surgery at Hôpital Necker-Enfants Malades in Paris. Ten patients in whom shunts were implanted at the referring hospital were excluded. The medical records and neuroimaging studies obtained in the remaining 196 patients were reviewed. These patients were categorized into three groups: 67 patients with hydrocephalus on admission in whom endoscopically guided third ventriculostomy was performed prior to tumor removal (Group A); 82 patients with hydrocephalus in whom preliminary third ventriculostomy was not performed and who were managed in a "conventional way" (Group B); and 47 patients without ventricular dilation on admission (Group C).

There was no significant difference between Group A and Group B patients with respect to age at presentation, evidence of metastatic disease, degree of tumor resection, or follow up. In the patients in Group A, however, more severe hydrocephalus was present $(\mathrm{p}<0.01)$. Patients in Group $\mathrm{C}$ were, in this respect, different from the other two groups.

Ultimately, only four patients $(6 \%)$ in Group A as compared with 22 patients $(27 \%)$ in Group B (p= 0.001 ) had progressive hydrocephalus requiring treatment following removal of the posterior fossa tumor. Sixteen patients (20\%) in Group B underwent insertion of a ventriculoperitoneal shunt, which is similar to the incidence of this procedure reported in the literature and significantly different from that in Group A ( $\mathrm{p}<0.016)$. The other six patients in Group B $(6 \%)$ were treated by endoscopically guided third ventriculostomy after tumor removal. In Group C, two patients (4\%) with postoperative hydrocephalus underwent endoscopically guided third ventriculostomy. 
In three of the patients who required placement of cerebrospinal fluid shunts several episodes of shunt malfunction occurred; these were ultimately managed by performing endoscopic third ventriculostomy and definitive removal of the shunt.

There were no cases of death and four cases of transient morbidity associated with the ventriculostomy.

Third ventriculostomy is feasible even in the presence of posterior fossa tumors (including brainstem tumors). When performed prior to posterior fossa surgery, it significantly reduces the incidence of postoperative hydrocephalus. Furthermore, it provides a valid alternative to the placement of permanent shunts in cases in which hydrocephalus develops following posterior fossa surgery, and it may negate the need for the shunt in cases in which the shunt malfunctions.

Although the authors acknowledge that the routine application of third ventriculostomy in selected patients may result in a proportion of patients undergoing an "unnecessary" procedure, they believe that because of patients' less complicated postoperative course, the low morbidity rate, and the high success rate of third ventriculostomy, further investigation of this protocol is warranted.

\section{Key Words * pediatric patient * posterior fossa tumor * hydrocephalus * treatment * third ventriculostomy * endoscopy}

The management of hydrocephalus associated with posterior fossa tumors in children has always been problematic: although the majority of patients will not require permanent cerebrospinal fluid (CSF) diversion, those patients who do so appear to suffer a more complicated postoperative course[15] and are subject to the well-recognized problems associated with these devices. Although in the past placement of a shunt in these patients was often considered to be appropriate preoperative treatment,[2] technological advances and changes in the availability of neuroimaging devices have allowed for earlier diagnosis. Consequently, most pediatric neurosurgeons today utilize a combination of corticosteroid drug therapy, early surgery, and external ventricular drainage when necessary.

In the literature, approximately one third of patients overall with hydrocephalus will eventually require a shunt.[15] The factors associated with required shunt placement have been retrospectively analyzed[6,15] and include young age ( $<10$ years of age); midline tumors; incomplete tumor resection; CSF infection; and persistent pseudomeningocele.

Internal CSF diversion is an appealing management option because of the obstructive nature of the hydrocephalus associated with these tumors. [4,11] Despite this, third ventriculostomy remains underutilized because of a number of concerns. Although the obstructive hydrocephalus may be controlled prior to tumor resection, there is a paucity of literature regarding the site of interference of CSF dynamics after tumor removal, thus the long-term efficacy of the procedure is questionable. More important, there is the question of safety in performing a third ventriculostomy in the presence of a large posterior fossa mass. The fact that this procedure may be unnecessary in some patients emphasizes the need for attaining low morbidity rates. In this paper we report the findings of a retrospective study aimed at addressing some of the attendant concerns of this procedure.

\section{CLINICAL MATERIAL AND METHODS}

Two hundred six patients with posterior fossa tumors were admitted to the pediatric neurosurgical department at the Hôpital Necker-Enfants Malades between October 1, 1993, and December 31, 1997. 
Ten patients in whom shunts had been placed at the referring hospital were excluded from the present study. Of the remaining 196 patients, 186 underwent open posterior fossa surgery to achieve gross-total tumor removal. In the remaining 10 patients assessment was performed and/or a biopsy sample was obtained.

These patients, although not randomized, fell into three groups. In the first group (Group A) the patients presented with symptoms and/or signs of intracranial hypertension, with ventricular dilation evidenced on the initial computerized tomograpy (CT) scan or magnetic resonance (MR) image. In these patients an endoscopically guided third ventriculostomy was performed on admission according to the technique previously described.[21] Surgery to resect the tumor was then performed when convenient. In the second group (Group B) the patients presented with symptoms similar to those in Group A; however, in these patients third ventriculostomy was not performed prior to resection of the posterior fossa tumor predominantly because personal surgical preference among the five staff pediatric neurosurgeons. These patients were treated in a "conventional" fashion (that is, steroid therapy, early surgery, and ventricular drainage when needed). Group $\mathrm{C}$ consisted of those patients in whom no evidence of ventricular dilation had been demonstrated on initial CT scanning.

The hospital records and imaging studies of the 196 patients were retrospectively reviewed with special attention being paid to age at the time of treatment; treatment of hydrocephalus (both type and timing of treatment); complications associated with treatment of hydrocephalus; operative position, site of tumor (midline, brainstem, involving the fourth ventricle, hemispheric, or cerebellopontine angle); degree of tumor resection at the time of posterior fossa surgery (that is, complete, subtotal, partial, or biopsy); type of tumor; postoperative course (that is, CSF leak, pseudomeningocele, and treatment); follow-up course; and shunt placement or removal during the follow-up period.

On imaging studies the degree of ventricular dilation was assessed and graded as follows: normal, mild, moderate, or severe, according to the same clinician's review of the initial CT scan. All patients underwent MR imaging prior to the third ventriculostomy and tumor removal. The site of the tumor as well as evidence of metastatic disease were documented. In all patients CT scanning with and without administration contrast material was performed within 24 hours of tumor removal, and these studies were used to grade the degree of tumor resection (in complete and subtotal resections no areas of contrast enhancement were demonstrated). The resection was considered to be partial when evidence of an area of contrast enhancement was revealed on the postoperative CT scan. In the initial phase of the study, patients undergoing third ventriculostomy were examined using cine MR imaging both before and after tumor surgery.

\section{RESULTS}

\section{Group A}

There were 67 patients in Group A, and their mean age at presentation was 6.6 years (median age 5.7 years; Table 1). Imaging studies confirmed severe ventricular dilation in $60 \%$ of the cases (Table 1). Additionally in 16 patients (24\%) evidence of metastatic spread was demonstrated. 
TABLE 1

CHARACTERISTICS AND SEVERITV OF HYDROCEPHALUS IN PATIENTS WITH TUMORS OF THE POSTERIOR FOSSA

\begin{tabular}{|c|c|c|c|}
\hline Characteristic & $\begin{array}{c}\text { Group A } \\
\text { (67 patients) }\end{array}$ & $\begin{array}{c}\text { Group B } \\
\text { (82 pabents) }\end{array}$ & $\begin{array}{c}\text { Group } C \\
\text { (97 patients) }\end{array}$ \\
\hline \multicolumn{4}{|l|}{ presentation } \\
\hline mean age (yrs) & & 7.0 & \\
\hline $\begin{array}{l}\text { age range } \\
\text { metastases }\end{array}$ & 46 days -15.1 yrs & 121 days-15.3 yrs & $1.1 \mathrm{yrs}-16 \mathrm{yrs}$ \\
\hline \multicolumn{4}{|c|}{$\begin{array}{l}\text { metastases } \\
\text { severity of hydrocephalus }(\%)\end{array}$} \\
\hline normal ventides & 0 & 0 & 100 \\
\hline mild & 9 & 36 & \\
\hline moderate & 31 & 38 & \\
\hline severe & 60 & 26 & \\
\hline
\end{tabular}

By definition, in all patients in Group A hydrocephalus was treated by an initial third ventriculostomy. There were no deaths and no permanent morbidity associated with this procedure. Four patients suffered immediate transient complications.

One patient suffered a perioperative episode of presumed "upward herniation" because of deterioration in consciousness and Parinaud's syndrome and underwent immediate removal of the posterior fossa tumor, with complete recovery. In the other three patients technical complications occurred. In one an incomplete third ventriculostomy was evidenced by the occurrence of ongoing symptoms and by a cine MR image that demonstrated no flow. The procedure was repeated, and resultant clinical resolution occurred. In the other two patients the third ventriculostomy procedure had to be aborted due to poor visibility: one secondary to a mild intraventricular hemorrhage and the other due to markedly xanthochromatic CSF. The patient in this latter case underwent immediate resection of the posterior fossa tumor and a repeated third ventriculostomy 10 days later. Thus 65 patients had a technically successful preliminary third ventriculostomy. All but one patient (98\%) experienced immediate symptomatic resolution. The mean time from ventriculostomy to posterior fossa surgery was 40 days (median 4 days, range $0-1533$ days).

Most of the tumors in Group A patients were midline. The details of tumor localization, and type and extent of resection are shown in Table 2. 


\begin{tabular}{|c|c|c|c|}
\hline \multicolumn{4}{|c|}{$\begin{array}{r}\text { TABLE } 2 \\
\text { LOCALEATION, TYPE, AND EXTENT OF TUMOF }\end{array}$} \\
\hline Characteristic & Group A & Group B & Group C \\
\hline \multicolumn{4}{|l|}{ tumor localization (\%) } \\
\hline midline & 67 & 56 & 21 \\
\hline cerebellar hemisphere & 14 & 23 & 32 \\
\hline Erainstem & 9 & 12 & 30 \\
\hline cerebellopontine angle & 10 & 9 & 17 \\
\hline \multicolumn{4}{|l|}{ tumor type (\%) } \\
\hline medullo blastoma & 46 & 35 & 13 \\
\hline epend ymoma & 24 & 11 & 17 \\
\hline astrocytoma & 21 & 39 & 42 \\
\hline ganglioglioma & 3 & 0 & 2 \\
\hline cavernoma & 1 & 2 & 3 \\
\hline PNET & 3 & 6 & 2 \\
\hline meningioma & 1 & 0 & $\overline{2}$ \\
\hline other & 1 & 7 & 19 \\
\hline \multicolumn{4}{|l|}{ extent of resection (\%) } \\
\hline total & 51 & 61 & 68 \\
\hline subtotal & 37 & 26 & 19 \\
\hline partial & 7 & 6 & 11 \\
\hline Eiopsy & 4 & 7 & 2 \\
\hline
\end{tabular}

All except one patient underwent an attempted gross-total resection. In 55 patients surgery was performed in the sitting position, whereas the remaining patients were in the ventral decubitus position. No substitute dural graft was used. The details of the postoperative complications are shown in Table 3. The mean follow-up period was 786 days (median 661 days, range 1-2003 days).

\begin{tabular}{|c|c|c|c|}
\hline \multicolumn{4}{|c|}{$\begin{array}{c}\text { TABLE } 3 \\
\text { POSTOPERATIVE COMPLICATIONS IN THE THREE GROUPS }\end{array}$} \\
\hline Postop Complication & Group A & Group B & Group C \\
\hline $\begin{array}{l}\text { no. of patients wi postop complications } \\
\text { mound dehiscenoe (CSF leak) } \\
\text { subdural collection (transient subdural shunt) } \\
\text { pseudomeningocele (extemal lum bar drainage) } \\
\text { epidural hematoma. } \\
\text { death }\end{array}$ & $\begin{array}{l}17(25 \% 67 \\
8(3) \\
4(2) \\
8(2) \\
1 \\
0\end{array}$ & $\begin{array}{l}31(38 \%) 82 \\
12(5) \\
4(1) \\
22(5) \\
5 \\
2\end{array}$ & $\begin{array}{l}9(19 \%) 47 \\
5(1) \\
1(1) \\
7(4) \\
1 \\
1\end{array}$ \\
\hline
\end{tabular}

Four patients ultimately required the implantation of a ventriculoperitoneal (VP) shunt for progressive hydrocephalus. Shunt placement was required in one of these patients 19 days after undergoing removal of the posterior fossa tumor because of postoperative signs of intracranial hypertension and because of the personal preference of the on-call surgeon. In another patient enrolled in a clinical trial of presurgical chemotherapy and who did not undergo immediate tumor resection, a shunt was inserted 21 days after the third ventriculostomy procedure. In the remaining two patients the shunt was inserted 83 and 432 days, respectively, after tumor resection. In both of these patients rapidly progressive subarachnoid seeding of their primary tumor was demonstrated. Of the four patients, in one three episodes of shunt failure were associated with dorsal mesencephalic dysfunction. A new endoscopically guided third ventriculostomy was performed. The stoma was found to be obstructed; it was reopened, and the shunt was removed. 
Thirteen patients suffered tumor recurrence (10 in posterior fossa, two as intracranial seeding, and one as lumbar seeding). Only the two patients with intracranial seeding required a VP shunt (as discussed in the previous section). In one patient who experienced local recurrence of a posterior fossa ependymoma 4 years after undergoing preoperative third ventriculostomy, complete tumor removal, and chemotherapy, the tumor that recurred was completely excised. He subsequently presented with increasing ventricular size, pseudomeningocele, and gait disturbance while undergoing radiotherapy 8 months later. A cine MR image showed no flow through the stoma. An endoscopically guided third ventriculostomy was subsequently performed. At this time, the stoma was found to be obstructed and was reopened. Complete radiological and clinical resolution of the stoma was achieved.

\section{Group B}

There were 82 patients in Group B. The mean age at surgery was 7 years (median age 6.3 years; Table 1). On imaging studies, hydrocephalus was designated as moderate in $38 \%$ and severe in $26 \%$ of the cases (Table 1). In sixteen patients (20\%) evidence of metastatic spread was demonstrated.

The majority of the tumors in Group B patients were midline. The details of tumor localization and type and extent of resection are shown in Table 2. Gross-total resection was achieved in 71 of 76 patients in whom it was attempted. In six patients only a biopsy sample was obtained. The mean follow-up period was 744 days (median 624 days, range 1-1697 days).

Sixty-six patients underwent surgery while in the sitting position, whereas the remaining patients were in the ventral decubitus position. No substitute dural graft was used. External ventricular drainage was used in nine patients prior to tumor removal, in six at the time of surgery, and in six patients in the postoperative period. Emergency evacuation of an extradural hematoma was required in five cases. Three of these hematomas occurred at the site of the external ventricular drain, and two were located in the posterior fossa. All of these patients had undergone surgery while in the sitting position. Two patients died in the early postoperative period: one of acute intracranial hypertension 48 hours after incomplete removal of a fourth ventricular medulloblastoma. In the second patient surgery was performed after placement of an emergency external ventricular drain failed to reverse the bilateral mydriasis present on admission. This patient subsequently died in the immediate postoperative period. The details of the postoperative complications are shown in Table 3.

Twenty-two patients (27\%) presented with symptoms and signs of intracranial hypertension and with radiological evidence of active hydrocephalus following posterior fossa tumor surgery. In 16 patients the resection was considered to have been radiologically complete. The extent of surgical resection was not statistically significant in predicting the incidence of postoperative hydrocephalus ( $p>0.02)$. In 18 patients the hydrocephalus was diagnosed within 2 months of surgery. In the four patients in whom the development of hydrocephalus was delayed $(6,7,7$, and 28 months, respectively), this occurred in the context of local recurrence or of subarachnoid seeding.

Sixteen patients were treated with a VP shunt, with a mean delay of 107 days (median 20 days, range 10-845 days) following posterior fossa surgery. Two patients subsequently experienced episodes of shunt malfunction. A third ventriculostomy procedure was performed in each of these two patients, curing the hydrocephalus and allowing for definitive removal of the shunt 5 months and 2 years, respectively, after shunt implantation.

In the remaining six patients the postoperative hydrocephalus was treated by endoscopically guided third 
ventriculostomy, with a mean delay of 16 days (median 7 days, range 3-42 days) after posterior fossa surgery. In two patients evidence of tumor spread in the subarachnoid spaces was revealed at the time of third ventriculostomy. In all cases complete resolution of hydrocephalus was observed following the procedure, and the patients remained symptom free during the follow-up period (mean 1.4 years).

\section{Group C}

There were 47 patients in Group $C$ with the mean age at surgery of 7.8 years. Hydrocephalus was not observed in these patients on admission, the tumor being located, in most cases, in the cerebellar hemispheres or in the brainstem (Tables 1 and 2).

Gross-total removal of the tumor was attempted in 46 patients, and in one patient only a biopsy sample was obtained. The results of surgical resection are shown in Table 2. No foreign dural substitute was used. One patient died of a sudden, unexplained cardiac arrest 6 days after partial removal of a fourth ventricular medulloblastoma. The details of the postoperative complications are shown in Table 3 . The mean follow up was 756 days (median 638 days, range 1-1650 days).

Two patients (4\%) presented with symptoms and sign of intracranial hypertension and with radiological evidence of active hydrocephalus 3 months and 5 months, respectively, after posterior fossa surgery. They underwent with endoscopically guided third ventriculostomy and remained symptom free during the follow-up period (mean 2 years). No VP shunts were placed postoperatively in this group.

\section{Analysis of Results}

Significantly worse hydrocephalus on admission was demonstrated in Group A patients $(\mathrm{p}<0.01$, chi-square test). Although there were no significant differences between Groups A and B for all the other risk factors examined, in Group B patients a significantly higher incidence of postoperative hydrocephalus that required surgical treatment was demonstrated $(\mathrm{p}=0.001)$ and significantly more VP shunts were inserted in this group during the study period $(\mathrm{p}<0.02$, Fisher's exact test).

We observed fewer immediate postoperative complications in the patients in Group A compared with those in Group B (25\% and 38\%, respectively). We observed (but were not able to quantify) a smoother postoperative course and a faster recovery in patients in Group A compared with those in Group B.

\section{DISCUSSION}

The association of posterior fossa tumors with hydrocephalus, each a potentially lethal condition,[19,24] necessitates urgent surgical treatment in these seriously ill children. In the 1960s, when children presented in a poor clinical state due to a delayed diagnosis,[15] the routine use of preoperative shunts[1,10] significantly reduced the overall morbidity and mortality rates.[2]

Improvements in the availability and type of neuroimaging devices has enabled a markedly earlier diagnosis to be made. This, coupled with a growing awareness of "common" complications associated with ventricular shunting and rarer complications more specific to this patient population (such as upward herniation, hemorrhage of the tumor, and peritoneal seeding of the intracranial tumor), caused neurosurgeons to question the need for routine shunt insertion. $[8,9,14,16]$

The result was a move toward a more expectant policy with patients undergoing corticosteroid medication therapy, early surgery, and external ventricular drain when needed.[17,20,23] Although theoretically appealing, this protocol was not without risk. An external ventricular drainage system used 
in this setting was not without risk of view of causing infection (an incidence of $4.9 \%$ according to a report by Schmid and Seiler[22]) and upward herniation or hemorrhage, the latter of which is underscored by the five patients in the present series who developed subsequent to ventricular drainage, intracranial hematoma that required surgical evacuation. Of more concern, the more recent studies of this protocol have shown that ultimately 17 to $40 \%[6,7,15,18,19,22]$ of children who harbor tumors in the posterior fossa have uncontrolled hydrocephalus and require shunt placement during the postoperative period; Culley and colleagues[6] and Lee[15] have reported that this predominantly occurred within the 1 st postoperative month. In adopting this expectant policy, those patients who ultimately require a shunt are thereby placed at risk of developing intracranial hypertension, an increased rate of CSF leakage, and pseudomeningocele formation, [6,15] prolonged hospitalization, and a higher rate of pseudobulbar palsy.[15] For these patients, arguably, we must do better.

Consequently, several authors have attempted to identify, through retrospective analysis, those patients most at risk of requiring placement of a shunt postoperatively and have identified several possible factors. [6,7,15] Younger age at diagnosis has been shown to be significant by most $[3,6,15,18]$ but not all authors.[7] The severity of hydrocephalus prior to resection of the tumor seems to be a predictor of shunt requirement in patients with medulloblastoma[15] but not in those with other types of tumors.[7,18] Midline localization,[6,15] incomplete tumor removal,[6] the use of substitute dural grafts during closure,[6] and higher Chang stages in patients with medulloblastoma[15] are also considered by several authors to be significant risk factors for postoperative shunts. On the contrary tumor size and the use of external ventricular drainage[6] do not appear to have a direct prognostic effect.[22]

In the present series, despite the lack of randomization, Group A and Group B patients were similar in terms of all the aforementioned positive risk factors. In addition, in the patients in Group A significantly more hydrocephalus was shown on admission. The patients in Group $\mathrm{C}$ were, by definition, different. The incidence of shunt insertion (16 [20\%] 82) in Group B was in keeping with that found in the literature and would have been even higher if all the patients with postoperative hydrocephalus had undergone insertion of a VP shunt (six patients having been treated with third ventriculostomy). On the contrary, in patients in Groups A and C a significantly lower rate of postoperative hydrocephalus was found. For Group C, this probably means that, in the present study, it is possible to define, retrospectively, a group of patients who harbored posterior fossa tumors in whom hydrocephalus was not demonstrated on admission and for whom there is a low risk of developing postoperative hydrocephalus. Of more interest is those patients in Group A in whom it is apparent that endoscopically guided third ventriculostomy had a curative effect on intracranial hypertension preoperatively and, conceivably, a prophylactic effect in the prevention of the development of hydrocephalus postoperatively. This is plausible when one considers the pathophysiology of hydrocephalus in these patients.

Preoperatively the development of hydrocephalus relates to the nature and localization of these tumors. Tumors arising from within the fourth ventricle fill and obstruct the ventricular cavity and its foraminal outlets. Tumors of the cerebellar hemisphere, on the other hand, induce severe anatomical distortion of the fourth ventricle and subsequent occlusion of the CSF pathways. Subarachnoid seeding may be observed at diagnosis in patients harboring malignant tumors that favor impairment of the subarachnoid pathways.

Logically, the complete removal of the tumor reestablishes a communication between the fourth ventricular cavity and the subarachnoid spaces, restoring a physiological CSF circulation and allowing resolution of hydrocephalus. Nevertheless, in the immediate postoperative period, acute increase of 
resistance on the CSF circulation and resulting intracranial hypertension may be induced by two factors; the surgery-induced subarachnoid hemorrhage and the presence of cerebellar swelling. Later on, one fourth to one third of the patients remain hydrocephalic. The use rate of third ventriculostomy in this series successfully confirmed the obstructive nature of postoperative hydrocephalus in all cases. The secondary development of adhesions at the level of the fourth ventricular outlets and the adjacent cisterns may permanently alter CSF hydrodynamics. This hypothesis is underscored by the higher incidence of postoperative hydrocephalus after vermis splitting and cisterna magna opening to gain access to the tumor. Finally, hydrocephalus may develop several months or years following the primary surgery, at the time of a local recurrence or of subarachnoid spread.

The obstructive nature of hydrocephalus in cases of posterior fossa tumors that are localized to the level of the fourth ventricular outlets both pre- and postoperatively provides the rational basis for the curative effect of endoscopically guided third ventriculostomy. The significant postoperative reduction in the rate of hydrocephalus seen in our patients who underwent preliminary third ventriculostomy when compared with those in whom perioperative control of the hydrocephalus was obtained via ventricular drainage suggests that an additional prophylactic effect is in evidence. Preoperative normalization of CSF hydrodynamics seems to decrease the risk of developing of permanent postoperative impairment of the CSF circulation.

Rationally, third ventriculostomy would appear to be the procedure of choice in the postoperative control of hydrocephalus in patients with posterior fossa tumors. However, there is a noticeable dearth of information regarding this in the literature. Jones et al.,[12] have reported on the use of third ventriculostomy in the postoperative period in two patients, one procedure performed 3 days following surgery for recurrent medulloblastoma, which was successful, and the second was performed 3 years after the patient underwent excision of an ependymoma and radiotherapy, which proved technically impossible to perform due to hemorrhage.

In the present series endoscopically guided third ventriculostomy was performed in eight cases following resection of a posterior fossa tumor (six in Group B and two in Group C) and successfully managed to control the hydrocephalus in all cases, even in the presence of subarachnoid seeding of the tumor that was evident at the time of the procedure. Furthermore, three patients who had undergone postoperative shunt placement and in whom subsequent shunt malfunction occurred were able to have had their hydrocephalus successfully treated by third ventriculostomy.[5]

The low morbidity and mortality rates associated with this technique reaffirm its valid use as an alternative to placement of a ventricular shunt in these individuals. What remains controversial, however, is the routine application of third ventriculostomy prior to posterior fossa tumor resection in those patients presenting with hydrocephalus. This concept, however, is not new.

In the past, third ventriculostomy has been used intermittently in the management of hydrocephalus associated with posterior fossa lesions.[12,13] In 1987 Jones and coworkers[12] appear to have been the first to have attempted endoscopically guided third ventriculostomy prior to posterior fossa tumor removal. They reported on one patient with a medulloblastoma in whom the procedure was performed 6 days prior to tumor removal. Although the procedure was successful at controlling the intracranial hypertension, the patient experienced increased ataxia. We did not observe this phenomenon in any of our patients. Hopf, et al.,[11] have recently reported on17 cases of endoscopically guided third ventriculostomy in patients harboring posterior fossa neoplasms, and in these cases the hydrocephalus 
was successfully managed in $13(76 \%)$. No details were given on the modality or the timing of failure.

What makes the present series unique was the systematic application of third ventriculostomy prior to definitive posterior fossa surgery. This protocol was instituted in an attempt to reduce the not insignificant rate of postoperative hydrocephalus with its associated, significant incidence of morbidity uncontrolled intracranial hypertension as well as placement of ventricular shunt systems. However, in endorsing such a protocol it is apparent that a proportion of patients will undergo an "unnecessary" procedure. How can this be justified?

First, third ventriculostomy in our hands appears to be safe. There were no deaths associated with our technique and only four cases of transient morbidity, three of which were technical failures. Only one patient was neurologically worse following the procedure, the cause of which we can only speculate to have been the result of upward herniation that resolved with immediate posterior fossa decompression. The procedure, in which we used an atraumatic balloon dilation and slow irrigation when required,[21] was no more difficult technically than that for congenital aqueductal stenosis. Theoretically, the tumor bulk may displace the brainstem forward and obliterate the prepontine cistern, thus making third ventriculostomy impossible. Although initially wary of this, we have not found it to be a problem in our experience. In fact, tumors of the posterior fossa tend to elevate the brainstem at the same time that they push it forward. This means that the floor of the third ventricle is displaced largely above the posterior clinoid thus making room for the fenestration (Fig. 1).
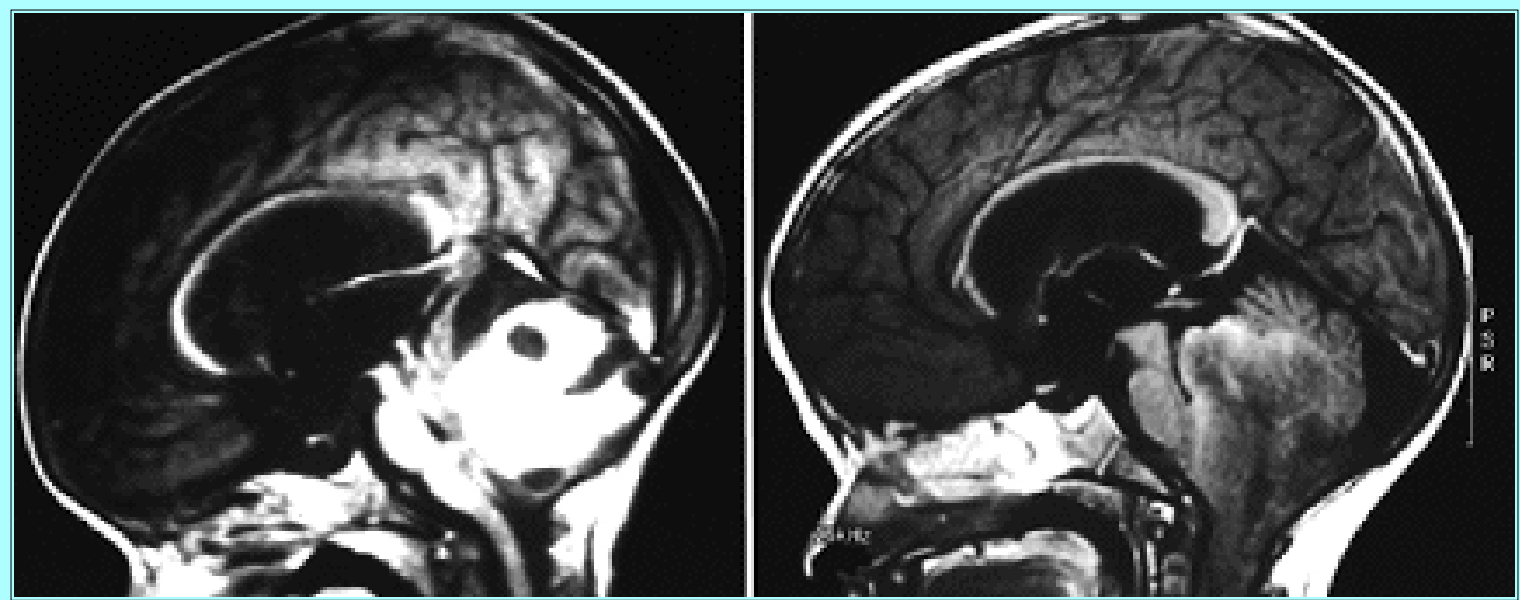

Fig. 1. Sagittal $T_{1}$-weighted $M R$ images revealing brainstem displacement induced by a posterior fossa tumor. The brainstem is pushed forward on the clivus but also upward by the tumor, elevating the posterior part of the floor of the third ventricle. Upper: Image obtained in a 5-year-old girl with a large vermian medulloblastoma. Lower: Image obtained in a 3 -year-old boy with an exophytic brainstem glioma.

Second, the preoperative control of the hydrocephalus may in fact provide benefit to these patients. The improved morbidity and mortality rates reported in the earlier studies after the introduction of ventricular drainage is indubitably a reflection of the poor clinical state of the child at diagnosis.[2] However, given the smoother postoperative course observed in our patients treated with preliminary third ventriculostomy and the reduction in postoperative complications, it is the management of the hydrocephalus itself that may be contributory.

Thus, third ventriculostomy in our series is a safe therapy that is a valid alternative in the treatment of postoperative hydrocephalus. Further when used prior to posterior fossa tumors it reduces the risk of 
postoperative hydrocephalus and, in so doing, may reduce the incidences of morbidity and mortality in these patients. The systematic use of third ventriculostomy prior to definitive surgery in patients with hydrocephalus on admission warrants further investigation.

\section{CONCLUSIONS}

The preliminary results from this retrospective study on a significant number of patients show that endoscopically guided third ventriculostomy is a feasible, safe method of treating hydrocephalus associated with posterior fossa tumors. When performed before posterior fossa surgery, it had both a curative effect on intracranial hypertension prior to tumor removal and a prophylactic effect by preventing the development of hydrocephalus after tumor removal. When performed after posterior fossa surgery, it successfully resolved the hydrocephalus in all cases. The significantly lower incidence of postoperative hydrocephalus in the group treated by third ventriculostomy prior to tumor removal is highly encouraging when compared with our conventionally treated group and with the literature and offers a consistent base for future prospective controlled studies to confirm these findings.

\section{References}

1. Abraham J, Chandy J: Ventriculo-atrial shunt in the management of posterior fossa tumours. Preliminary report. J Neurosurg 20:252-253, 1963

2. Albright L, Reigel DH: Management of hydrocephalus secondary to posterior fossa tumors. J Neurosurg 46:52-55, 1977

3. Allen JC, Epstein F: Medulloblastoma and other primary malignant neuroectodermal tumors of the CNS. The effects of patients' age and extent of disease on prognosis. J Neurosurg 57:446-451, 1982

4. Cinalli G, Sainte-Rose C, Chumas P, et al: Failure of third ventriculostomy in the treatment of aqueductal stenosis in children. J Neurosurg 90:448-454, 1999

5. Cinalli G, Salazar C, Mallucci C, et al: The role of endoscopic third ventriculostomy in the management of shunt malfunction. Neurosurgery 43:323-1329, 1998

6. Culley DJ, Berger MS, Shaw D, et al: An analysis of factors determining the need for ventriculoperitoneal shunts after posterior fossa tumor surgery in children. Neurosurgery 34:402-408, 1994

7. Dias MS, Albright AL: Management of hydrocephalus complicating childhood posterior fossa tumors. Pediatr Neurosci 15:283-290, 1989

8. Epstein F, Murali R: Pediatric posterior fossa tumors: hazards of the "preoperative" shunt. Neurosurgery 3:348-350, 1978

9. Forrest DM, Cooper DGW: Complications of ventriculo-atrial shunts. A review of 455 cases. J Neurosurg 29:506-512, 1968

10. Hekmatpanah J, Mullan S: Ventriculo-caval shunt in the management of posterior fossa tumors. J Neurosurg 26:609-613, 1967

11. Hopf NJ, Grunert P, Fries G, et al: Endoscopic third ventriculostomy: outcome analysis of 100 
consecutive procedures. Neurosurgery 44:795-806, 1999

12. Jones RF, Stening WA, Brydon M: Endoscopic third ventriculostomy. Neurosurgery 26:86-92, 1990

13. Kelly PJ, Goerss S, Kall BA, et al: Computed tomography-based stereotactic third ventriculostomy: technical note. Neurosurgery 18:791-794, 1986

14. Keucher TR, Mealy J Jr: Long-term results after ventriculoatrial and ventriculoperitoneal shunting for infantile hydrocephalus. J Neurosurg 50:179-186, 1979

15. Lee M, Wisoff JH, Abbott R, et al: Management of hydrocephalus in children with medulloblastoma: prognostic factors for shunting. Pediatr Neurosurg 20:240-247, 1994

16. McLaurin RL: Disadvantages of the preoperative shunt in posterior fossa tumors. Clin Neurosurg 30:286-294, 1983

17. Muszynski CA, Laurent JP, Cheek WR: Effects of ventricular drainage and dural closure on cerebrospinal fluid leaks after posterior fossa tumor surgery. Pediatr Neurosurg 21:227-231, 1994

18. Papo I, Caruselli G, Luongo A: External ventricular drainage in the management of posterior fossa tumors in children and adolescents. Neurosurgery 10:13-15, 1982

19. Raimondi AJ, Tomita T: Hydrocephalus and infratentorial tumors. Incidence, clinical picture, and treatment. J Neurosurg 55:174-182, 1981

20. Rappaport ZH, Shalit MN: Perioperative external ventricular drainage in obstructive hydrocephalus secondary to infratentorial brain tumours. Acta Neurochir 96:118-121, 1989

21. Sainte-Rose C: Third ventriculostomy, in Manwaring KH, Crone KR (eds): Neuroendoscopy. New York, Mary Ann Liebert, 1992, Vol 1, pp 47-62

22. Schmid UD, Seiler RW: Management of obstructive hydrocephalus secondary to posterior fossa tumors by steroids and subcutaneous ventricular catheter reservoir. J Neurosurg 65: 649-653, 1986

23. Shalit MN, Ben Ari Y, Eynan N: The management of obstructive hydrocephalus by the use of external continous ventricular drainage. Acta Neurochir 47:161-172, 1979

24. Stein BM, Tenner MS, Fraser RA: Hydrocephalus following removal of cerebellar astrocytomas in children. J Neurosurg 36:763-768, 1972

Manuscript received September 2, 1999.

Accepted in final form September 21, 1999.

Address reprint requests to: C. Sainte-Rose, M.D., Service de Neurochirurgie, Hôpital Necker-Enfants Malades, 149, rue de Sèvres, Paris 75743, Cedex 15, France. 